\title{
Blind Jealousy? Romantic Insecurity Increases Emotion-Induced Failures of Visual Perception
}

\author{
Steven B. Most, Jean-Philippe Laurenceau, Elana Graber, Amber Belcher, and C. Veronica Smith \\ University of Delaware
}

\begin{abstract}
Does the influence of close relationships pervade so deeply as to impact visual awareness? Results from two experiments involving heterosexual romantic couples suggest that they do. Female partners from each couple performed a rapid detection task where negative emotional distractors typically disrupt visual awareness of subsequent targets; at the same time, their male partners rated attractiveness first of landscapes, then of photos of other women. At the end of both experiments, the degree to which female partners indicated uneasiness about their male partner looking at and rating other women correlated significantly with the degree to which negative emotional distractors had disrupted their target perception during that time. This relationship was robust even when controlling for individual differences in baseline performance. Thus, emotions elicited by social contexts appear to wield power even at the level of perceptual processing.
\end{abstract}

Keywords: emotion-induced blindness, attention, close relationships, threat sensitivity, visual awareness

"It is not love that is blind, but jealousy."

—Lawrence Durrell, Justine, 1957

It is widely recognized that social relationships impact our moods, behaviors, and health (Reis, Collins, \& Berscheid, 2000), and insights into their power to do so are as much the domain of poets and philosophers as they are of psychological scientists. However, some have argued that psychological science has yet to consider fully the boundaries at which close relationship contexts can influence basic cognitive and perceptual processes (Reis \& Collins, 2004). Can fluctuations in perceived social context affect us so deeply as to influence even our visual processing of the world?

This question is not far-fetched: visual awareness of items in the environment depends largely on our ability to direct attention to them (Chun \& Marois, 2002; Mack \& Rock, 1998; Most, Scholl, Clifford, \& Simons, 2005; Most et al., 2001; Neisser \& Becklen, 1975; Simons \& Chabris, 1999), and we tend to prioritize emotional stimuli to such a degree that doing so can impair visual awareness of nearby nonemotional information (Most \& Jungé, 2008; Most et al., 2007; Most, Chun, Widders, \& Zald, 2005). The degree to which emotionally significant items capture attention seems to be modulated by one's mood and temperament (e.g., Fox,

Steven B. Most, Jean-Philippe Laurenceau, Elana Graber, Amber Belcher, and C. Veronica Smith, Department of Psychology, University of Delaware.

Preparation of this article was facilitated by a grant from the National Institute of Mental Health to J-P Laurenceau (K01MH64779). Thanks to Matt Shaffer, Patrick Ewell, Ben Hadden, Elizabeth Sullo, and Lauren Pulinka for their help in running participants.

Correspondence concerning this article should be addressed to Steven B. Most or Jean-Philippe Laurenceau, Department of Psychology, University of Delaware, 108 Wolf Hall, Newark, DE 19716-2577. E-mail: most@psych.udel.edu or jlaurenceau@psych.udel.edu
Russo, Bowles, \& Dutton, 2001), and thus, to the degree that social contexts influence one's affective state, it is conceivable that they impact the processes involved in visual awareness as well.

Close relationships are one of the primary contexts for the experience of emotion (Berscheid, 1983) and recent evidence from the neuroimaging literature suggests that social context modulates activity within neural regions typically responsive to threat. For example, while in a functional MRI scanner, married women viewed cues that either were or were not indicative of potentially imminent electric shock, and while viewing these cues they either held the hand of their husband, the hand of a male stranger, or no hand at all (Coan, Schaefer, \& Davidson, 2006). Not surprisingly, brain regions associated with response to threat showed enhanced activation following cues predicting a shock, but-importantlythreat-related activity in such regions was diminished when the participant held her husband's or a stranger's hand. Moreover, holding one's husband's hand was associated with greater attenuation of threat-related brain activation than holding a stranger's hand, and the degree to which holding one's husband's hand attenuated such activation was correlated with self-reported marital satisfaction.

If the presence of social support within the context of a romantic relationship aids in the down-regulation of affective reactivity to emotionally aversive stimuli, it could be that a perceived threat to the relationship would have the opposite effect, inducing-perhaps by increasing anxiety or unease-a heightened state of sensitivity to emotionally aversive cues. If so, given that reflexive attention to emotional stimuli can temporarily impair conscious perception (Most et al., 2005), it would raise the intriguing possibility that fluctuations in security regarding one's romantic relationship can literally affect how one sees the world.

We investigated this possibility in two studies. We recruited heterosexual romantic couples and administered a rapid attention task to the female partner, in which she searched for a single target within a sequence of fleeting images, all the while trying not to be 
distracted by a neutral or emotional picture that could appear. Typically, in this task, people have more difficulty reporting the target when an emotional distractor appears before or immediately after the target than when a neutral distractor appears (Most \& Jungé, 2008; Most et al., 2005). In a manipulation of perceived relationship threat (following from a previous study; Simpson, Ickes, \& Blackstone, 1995), the female partner performed this task while her male partner first rated the attractiveness of landscapes and then while he rated the attractiveness of other women who presumably were romantically accessible. Because we assumed that the relationship threat manipulation-which was the critical manipulation of interest-would not be equally effective for all participants, the female partner was asked at the end of the experiment to report how uneasy she was about the fact that her partner had been rating other women, and the correlation between this measure and emotion-induced blindness during the time that he was doing so served as our primary focus of analysis. Meanwhile, trials administered during the time that the male partner rated landscapes ensured that all participants received substantial practice before the relationship threat manipulation, and these trials were also included in the analyses to ensure that the primary correlations of interest stemmed from the manipulation itself rather than solely from baseline, trait-related individual differences (e.g., whereby participants who felt most threatened by the manipulation might also happen to be sensitive to emotional distractors generally). To further assess whether potential links between levels of discomfort caused by this relationship threat and temporary perceptual lapses might be accounted for by mere trait level individual differences, participants also completed measures of global threat sensitivity and of relationship-specific threat sensitivity.

\section{Experiment 1}

\section{Method}

Participants. Twenty-five heterosexual romantic couples were recruited from an ongoing study on close relationships. Either one or both of the partners was enrolled in an introductory psychology class and received extra course credit for their participation in this experiment. The average age of the female partners was 19.2 years, and the average age of the male partners was 20.11 years. Average length of relationship in months was 10.96 (ranging from 1 to 38 months). All participants gave informed consent.

Materials and procedure. Before the laboratory session, participants completed a demographics background survey and a brief set of paper-and-pencil measures, including an established trait measure of global threat sensitivity (Behavioral Inhibition Scale, or BIS; Carver \& White, 1994) and of relationship threat sensitivity (RTS; Laurenceau, Kleinman, Kaczynski, \& Carver, in press). This latter measure consisted of five items such as "If I think something unpleasant is going to happen in my romantic relationship, I usually get pretty 'worked up' and upset" answered using a 4-point Likert scale of agreement. This scale has demonstrated good reliability, a unitary factor structure, and expected validity coefficients as a measure of relational threat sensitivity (Laurenceau et al., in press). The coefficient alphas for the RTS and the BIS in this sample were .61 and .77 , respectively.

Couples participated in the laboratory paradigm one couple at a time. Before beginning, the female partner received 12 practice trials: on each trial, 12 upright landscape and architectural photos flashed on a screen in rapid succession, and she was required to detect a single target within the stream: a landscape/architectural photo that was rotated 90-degrees clockwise or counterclockwise. The first several practice trials were presented at $200 \mathrm{~ms}$ per item, on the eighth trial this increased to $100 \mathrm{~ms}$ per item, and the last two trials were presented at $60 \mathrm{~ms}$ per item, which was to be the actual presentation speed during the experiment. At the end of the practice session, the female partner was informed that the streams of items during the experiment might also contain pictures of people or animals, some of which would be graphic, but that such pictures were always nontargets and should be ignored.

During the experiment, the female and male partners sat at computers in the same room, but they were several feet away from each other and separated by a curtain. The female and male partners performed different tasks from each other, which were coordinated so that they would end at roughly the same time. After they took their places, the experimenter stood within view of both partners and explained the first part of procedure: the female partner would perform the same task that she had just practiced while the male partner simply rated the attractiveness of landscapes. The experimenter then reminded the participants not to talk to each other during the experiment and left the room. Partway through the experiment, the female partner was instructed by the computer to retrieve the experimenter, who then stood again within view of both partners and explained the second part of the procedure: the female partner would continue with the same task, but the male partner - rather than rating the attractiveness of landscapeswould now be rating "the attractiveness of single women, some of whom are on campus here." The experimenter then left the room again. At the end of the experiment, the female partner was asked two questions, which appeared on the computer screen: (a) "When your partner was rating the attractiveness of other women, how uneasy did this make you?", and 2) "When your partner was rating the attractiveness of landscapes, how uneasy did this make you?" Both ratings were made using a Likert scale ranging from 1 (not uneasy at all) to 9 (very uneasy). After the experiment, the participants were fully debriefed, and it was additionally disclosed that the pictures of women rated by the male partner had been collected from the Internet and had no known association with the university.

Details of the female partner's task. Stimuli were color photographs: 56 emotionally aversive pictures, 56 emotionally neutral pictures, 56 scrambled versions of the aversive pictures, 210 upright landscape/architectural scenes, and 168 target images (84 landscape/architectural photos rotated 90-degrees to the left and right). Stimuli were $9.4-\mathrm{cm}$ wide, $7.1-\mathrm{cm}$ high, and were presented on a CRT monitor with a $99 \mathrm{~Hz}$ refresh rate via the Psychophysics Toolbox extensions for Matlab (Brainard, 1997; Pelli, 1997). Emotional and neutral pictures were drawn mostly from the International Affective Picture System (IAPS; Lang, Bradley, \& Cuthbert, 2001) and were supplemented by similar pictures from publicly available sources (see Most et al., 2005). Both the negative and the neutral pictures were of people or animals, with the negative images depicting graphic violence, distress, and medical trauma. The neutral pictures were balanced with the negative pictures for numbers of depictions of people and animals. Negative and neutral pictures had been rated for valence (i.e., negative vs. positive) and arousal (low vs. high) on a 9-point 
Likert scale by a separate group of individuals ( 6 men, 6 women). Negative pictures, relative to neutral pictures, were rated as more unpleasant (negative: $M=1.72, S D=.54$; neutral: $M=5.00$, $S D=.45 ; t(11)=13.41, p<.00001)$ and more arousing (negative: $M=6.07, S D=.68$; neutral: $M=3.18, S D=.55 ; t(11)=$ 4.86, $p<.001){ }^{1}$

Experiment trials consisted of a rapid serial visual presentation (RSVP) stream of 17 images. Except for two images, all were upright landscape/architectural photographs; the remaining two images consisted of the critical distractor and the target stimulus (see Figure 1). The critical distractor could be an emotional picture, a neutral picture, or an upright landscape/architectural photo that blended in with the rest of the stream (baseline); in each case, the critical distractor remained on-screen for $100 \mathrm{~ms}$ and, depending on the trial, it was the 4 th, 7 th, or 10 th item in the stream. The target stimulus was a landscape/architectural photo rotated 90 degrees to the left or right, which was always the 5 th item after the distractor. The 100-ms duration of the critical distractor was based on previous findings that even such rapid exposure is sufficient to elicit a general emotion-induced blindness effect (Most et al., 2005); all other items in the stream were presented for $60 \mathrm{~ms}$ to keep performance lower than ceiling levels even after substantial practice. At the end of each trial, participants pressed either the left-arrow key or the right-arrow key to indicate the rotation of the target. There were 42 trials during the first part of the experiment, while the male partner was rating landscapes, and 126 trials during the second part, while the male partner was rating other women.

Details of the male partner's task. During the first part of the experiment, the male partner rated the attractiveness of 22 landscape photos on a Likert scale ranging from 1 (very unattractive) to 9 (very attractive), and each photo was presented for $3 \mathrm{~s}$. During the second part of the experiment, he did the same for 66 photos of women, which had been collected from publicly available sources on the Internet.

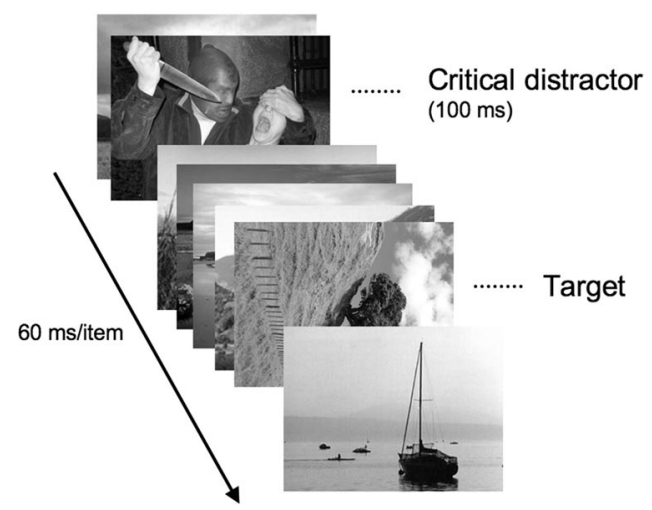

Figure 1. Schematic illustration of part of a trial. Participants' task was to detect the one rotated target in the stream and to indicate whether it had been turned clockwise or counterclockwise. The target always appeared as the 5th item following a critical distractor. In Experiment 1, critical distractors were either emotionally negative or neutral pictures or upright landscapes drawn from the same pool as most other items in the stream. In Experiment 2, critical distractors could also be erotic pictures. In both experiments, the critical distractors were presented for $100 \mathrm{~ms}$ and all other stream items were presented for $60 \mathrm{~ms}$.

\section{Results and Discussion}

Two couples were removed from the analyses because the female partner rated herself as being more uneasy when the male partner rated landscapes than when he rated other women, raising the possibility that these participants had misunderstood the question or the rating scale (both participants' ratings of unease when their partners rated landscapes were more than $2.5 S D$ s above the mean). An omnibus 3 (distractor valence: negative vs. neutral vs. baseline) $\times 2$ (partner's task: rate landscapes vs. rate other women) repeated measures ANOVA revealed a main effect of valence, $F(2,44)=16.78, p<.001, \eta_{\mathrm{p}}^{2}=.43$ : accuracy in the female partners' task was lower after a negative emotional distractor than after a neutral distractor, which in turn was lower than in the baseline condition. This was the case both when the male partner was rating landscapes (negative: $M=76.6 \%, S D=11.1 \%$; neutral: $M=82.3 \%, S D=11.4 \%$; baseline: $M=87.3 \%, S D=$ $14.0 \%$ ) and when he was rating other women (negative: $M=$ $85.3 \%, S D=8.4 \%$; neutral: $M=89.2 \%, S D=6.3 \%$; baseline: $M=92.3 \%, S D=4.8 \%$ ). Pairwise comparisons revealed that the performance following a negative emotional distractor was significantly worse than that following a neutral distractor regardless of the male partner's task (while partner rated landscapes: $t(22)=$ 2.66, $p=.01$; while partner rated other women: $t(22)=3.59, p=$ .002). Following from previous work (Most et al., 2005), we refer to this greater target perception impairment after an emotional distractor as "emotion-induced blindness." In addition, underscoring the importance of using the landscape-rating period to allow the female participants to grow accustomed to their task, there was a significant practice effect, as reflected in a main effect of partner's task, $F(1,22)=10.93, p=.003, \eta_{\mathrm{p}}^{2}=.33$ : that is, the female participants had higher accuracy in the second part and of the experiment, when her partner was rating other women, than in the first part of the experiment, when he was rating landscapes.

On average, when their male partners were rating the attractiveness of other women, female participants reported their level of uneasiness as 3.9 out of 9 , with uneasiness ranging from 1 to 8 across participants. (In contrast, their average level of uneasiness was 1.1 when their partners were rating landscapes, with a range from 1 to 2.) Notably, the degree to which female participants exhibited emotion-induced blindness while their partner rated the attractiveness of other women was strongly correlated with their self-rated level of unease about the fact that he was doing so, $r=$ $.45, p=.030$ (see Figure 2). ${ }^{2}$ In contrast, uneasiness about one's partner rating the attractiveness of other women did not correlate with degree of emotion-induced blindness elicited during the period when he had been rating landscapes, $r=-.001, p=.996$. To assess whether emotion-induced blindness during the time that one's partner was rating other women correlated with self-rated unease over and beyond trait-related individual differences, we conducted a partial correlation controlling for emotion-induced blindness during the landscape-rating condition. This partial cor-

\footnotetext{
${ }^{1}$ Two negative distractors were not included in these ratings. However, in the IAPS norms, their valence values were 1.7 and 2.35, and their arousal values were 7.03 and 6.91 , respectively.

2 This relationship was significant even when the two excluded couples were not removed from the analyses $(p<.05)$.
} 


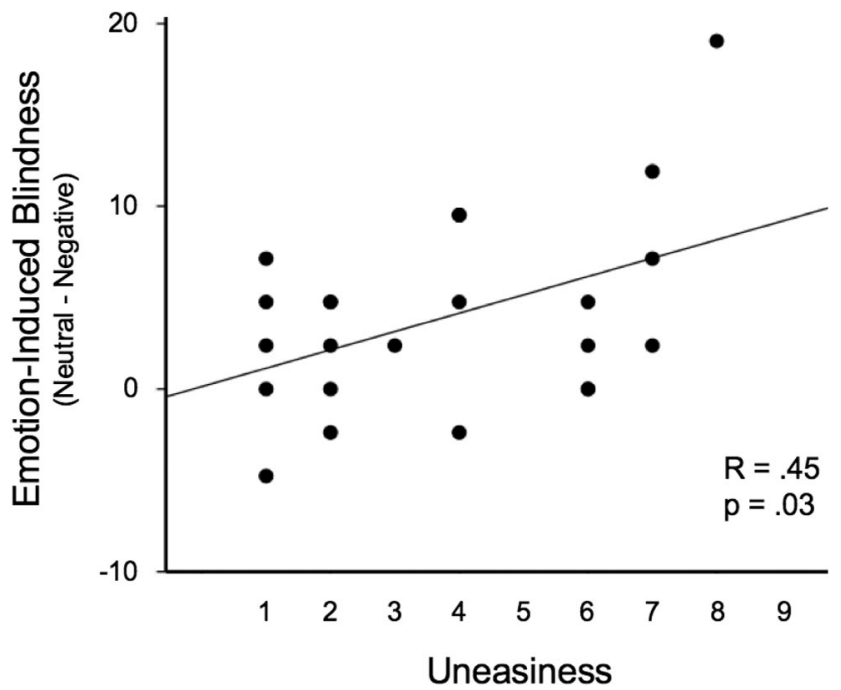

Figure 2. Data from Experiment 1. During the time that male partners were rating the attractiveness of other women, the degree to which female partners experienced emotion-induced blindness-that is, disruption of target perception caused by negative distractors over and beyond any disruption caused by neutral distractors-was significantly correlated with the degree to which they later rated themselves as having been uneasy about their male partner looking at other women.

relation revealed that this relationship remained significant, $r=$ $.45, p=.034$. To assess directly whether the correlation between unease and emotion-induced blindness was larger during the time that the male partner rated other women than when he rated landscapes, we compared these overlapping correlated correlation coefficients using Hotelling's $t$ (Meng, Rosenthal, \& Rubin, 1992). This difference was sizable but not statistically significant, $t(20)=$ $1.59, p=.128$, two-tailed.

We further addressed the possibility that individual differences in trait levels of global threat sensitivity or length of relationship could account for the above effects by conducting a regression analysis where BIS scores (Carver \& White, 1994) and relationship length (in months) were entered simultaneously with selfrated degree of unease about the male partner's task. In this model, unease remained a significant predictor of emotion-induced blindness $(\beta=.46, t=2.22, p=.039)$ while global threat sensitivity ( $\beta=-.01, t=0.07, n s)$ and relationship length $(\beta=-.08, t=$ $0.40, n s)$ were not. In addition, because the BIS serves as a global measure of trait anxiety, we also similarly examined whether individual differences in trait levels of relationship threat sensitivity (indexed via the RTS; Laurenceau et al., in press), specifically, could account for the above effects. As with the regression incorporating global BIS, unease remained a significant predictor of emotion-induced blindness $(\beta=.48, t=2.29, p=.03)$ while relationship threat sensitivity $(\beta=-.10, t=0.46, n s)$ and relationship length $(\beta=.08, t=0.38, n s)$ were not.

\section{Experiment 2}

Replication was a central goal of Experiment 2. However, we also took the opportunity to address two additional questions: (a) to what degree is heightened sensitivity to emotional distractors, as a function of the effectiveness of the relationship threat manipulation, specific to distractors of typically negative valence? And (b) do manipulations of relationship threat impact executive processes that might otherwise allow one to override emotion-induced blindness through strategic attentional tuning? Relevant to the first question, previous research has found that positive emotional stimuli can induce impairments in target perception as robustly as negative emotional stimuli can, as long as they elicit a large arousal response; indeed, erotic stimuli-which tend to be rated positively by both men and women (Bradley, Codispoti, Sabatinelli, \& Lang, 2001)—were found in one study to induce even more robust emotion-induced blindness than did graphic, emotionally negative stimuli (Most et al., 2007). One question, then, is whether discomfort with the fact that one's romantic partner is evaluating the attractiveness of other opposite sex individuals heightens sensitivity to these typically non-negative emotionally laden distractors that nonetheless contain content evocative of intimacy and potential relationship threat. In Experiment 2, the female partner's task included a set of erotic distractors as well as negative distractors.

Relevant to the second question, previous research has found that the degree to which emotionally negative distractors impair target perception can be modulated by one's attentional strategy, at least among non-trait-anxious individuals. In one study, for example, the targets in one condition were always pictures of a rotated building, whereas targets in another condition could be pictures either of rotated buildings or of rotated landscapes that contained no buildings (Most et al., 2005, Experiment 2). The rationale of the manipulation was that if people were given relatively specific information about their target, they should be able to "tune" their attention in such a way as to facilitate ignoring of emotional distractors. (This manipulation is intuitive if one imagines searching for a friend in a crowd: the search is more efficient if one knows more specifically what one's friend is wearing.) In that study, having more specific target information did not decrease emotion-induced blindness at all among people scoring high in a measure of "harm avoidance," which is associated with trait anxiety (Cloninger, Przybeck, \& Svrakic, 1991), but it completely eliminated the effect among those scoring low in harm avoidance (Most et al., 2005). In Experiment 2, we included a manipulation of target specificity to assess whether self-rated uneasiness would be associated with a weakened ability to use strategic tuning of attention to ignore emotional distractors.

In an additional modification to the design of Experiment 2, the number of trials completed while one's partner rated landscapes was the same as during the condition in which he rated other women. Although the landscape-rating condition served primarily as a control accounting for trait-like individual differences in emotion-induced blindness, this modification was important for addressing a possible shortcoming of the design of Experiment 1: in that experiment, the number of trials in the two conditions differed, and it was important to eliminate the possibility that this inequality drove differences in correlations with self-rated uneasiness.

\section{Method}

Participants. Twenty-seven male-female couples were recruited from an ongoing study on close relationships. Either one or 
both of the partners was enrolled in an introductory psychology class and received extra course credit for their participation in this experiment. The average age of the female partners was 19.35 years, and the average age of the male partners was 19.96 years. Average length of relationship was 10 months (ranging from 1 to 36 months). All participants gave informed consent.

Materials and procedure. The general procedure and paperand-pencil measures were the same as in Experiment 1. The coefficient alphas for the RTS and the BIS in this sample were .73 and .75, respectively. Critical distractors included the same emotionally negative and neutral sets of pictures, as well as a set of 56 erotic pictures of nude or seminude heterosexual couples. The target in all analyzed trials was a rotated picture of a building. Each of the eight blocks of 42 trials included 14 "filler" trials that were not analyzed, but which allowed the manipulation of attentional set: in the "specific attentional set" condition, the target on filler trials was a rotated building (thus ensuring that all targets in this condition were pictures of rotated buildings); in "nonspecific attentional set" blocks, it was a rotated landscape without a building. Filler trials contained no critical distractors. Blocks alternated by attentional set condition, with the specific attentional set condition always coming first; order of blocks was kept constant across participants to reduce noise that might otherwise obscure individual differences. ${ }^{3}$ Each female participant completed four blocks while her partner rated the attractiveness of landscapes and another four blocks while he rated the attractiveness of other women. Each block contained 7 negative, 7 neutral, 7 erotic, 7 baseline, and 14 filler trials.

\section{Results and Discussion}

Following the same criterion as in Experiment 1, one romantic couple was eliminated from the analyses because the female rated herself as experiencing more uneasiness when her partner rated landscapes than when he rated other women, raising the possibility that she had misunderstood the question or rating scale. An omnibus 4 (distractor valence: negative vs. neutral vs. erotic vs. baseline) $\times 2$ (attentional set: specific vs. nonspecific) $\times 2$ (partner's task: rate landscapes vs. rate other women) repeated measures ANOVA revealed a main effect of attentional set, $F(1,25)=$ $7.39, p=.012, \eta_{\mathrm{p}}^{2}=.23$, but contrary both to our predictions and with previous research (Most, Chun, Johnson, \& Kiehl, 2006; Most et al., 2005), accuracy was higher in the nonspecific attentional set condition $(M=86 \%, S D=9 \%)$ than in the specific attentional set condition $(M=83 \%, S D=7 \%)$. As noted in Footnote 3, this may have been because of a practice effect, as the experiment always began with the specific attentional set condition and ended with the nonspecific attentional set condition. A main effect of distractor valence also emerged, $F(3,75)=20.30, p<$ $.001, \eta_{\mathrm{p}}^{2}=.45$ : accuracy was lowest after erotic distractors $(M=$ $77 \%, S D=13 \%)$, next lowest after negative distractors $(M=$ $83 \%, S D=10 \%)$, next lowest after neutral distractors $(M=87 \%$, $S D=8 \%)$, and highest in the baseline condition $(M=91 \%, S D=$ $7 \%$ ). To test our main questions of interest-whether attentional set and self-rated unease predicted emotion-induced blindness-we created separate emotion-induced blindness indexes for the effect of negative distractors (accuracy in the neutral condition - accuracy in the negative condition) and of erotic distractors (neutral - erotic accuracy) for each attentional set condition during the time that the male partner rated other women. Pairwise comparisons revealed no significant effect of attentional set on emotion-induced blindness, whether induced by negative $(p>$ $.25)$ or erotic $(p>.90)$ distractors, so we collapsed across attentional set condition for the remainder of the analyses. As in Experiment 1, and again underscoring the importance of using trials in the landscape-rating condition as a means to get participants accustomed to the task, there was a main effect of partner's task, $F(1,25)=17.01, p<.001, \eta_{\mathrm{p}}^{2}=.41$ : the female participants had higher accuracy in the later part of the experiment, when her partner was rating other women, than in the earlier part of the experiment, when he was rating landscapes.

Most importantly, as in Experiment 1, the degree to which women reported unease about their partner rating the attractiveness of other women was significantly and inversely correlated with target detection accuracy following a negative distractor $(r=$ $-.42, p=.035)$, but not with accuracy following neutral $(r=$ $-.03, p=.88)$ or erotic $(r=-.22, p=.28)$ distractors or in trials containing no distractors $(r=-.12, p=.56)$. This translated into a significant correlation with emotion-induced blindness (i.e., the difference between accuracy in the emotional compare to neutral distractor trials) caused by negative distractors $(r=.55, p=.004$; see Figure 3 ) but not by erotic distractors $(r=.30, p=.14)$. Although the difference between these correlated correlation coefficients was not significant when tested directly with Hotelling's $t$, $t(23)=1.56, p=.132$, two-tailed (Meng et al., 1992), the correlation between emotion-induced blindness in the negative condition and unease was significant when controlling for emotion-induced blindness in the erotic condition, $r=.48, p=$ .015 (the reverse was not true). One possible explanation for the difference in strength between these correlations is that participants simply did not try as hard to ignore erotic distractors as they did negative emotional distractors. In a previous experiment, monetary performance-based incentives decreased emotion-induced blindness after negative emotional distractors but not after erotic distractors, suggesting that people either are truly less able to ignore erotic distractors than negative ones or that even large amounts of money fail to dissuade people from trying to glimpse representations of sexual conduct (Most et al., 2007). ${ }^{4}$

As in Study 1, we also controlled for the possibility that traitlike individual differences might account for the correlation between unease about one's partner rating other women and emotion-induced blindness caused by negative distractors during the time that he was doing so. A partial correlation, controlling for emotion-induced blindness during the landscape-rating condition, revealed that the effectiveness of the relationship threat manipulation predicted emotion-induced blindness while one's partner rated other women, over and beyond baseline levels of emotion-

\footnotetext{
${ }^{3}$ Note that this was a conservative ordering. Participants in previous studies have been shown to perform better in the specific attentional set condition (e.g., Most et al., 2005). However, by always starting with this condition in this experiment, it was the nonspecific attentional set condition that stood to benefit the most from accumulated practice.

${ }^{4}$ It is also worth noting that the erotic distractors might have been relevant to participants' frames of mind in unintended ways because of their depiction of intimacy within the context of a romantic relationship manipulation.
} 


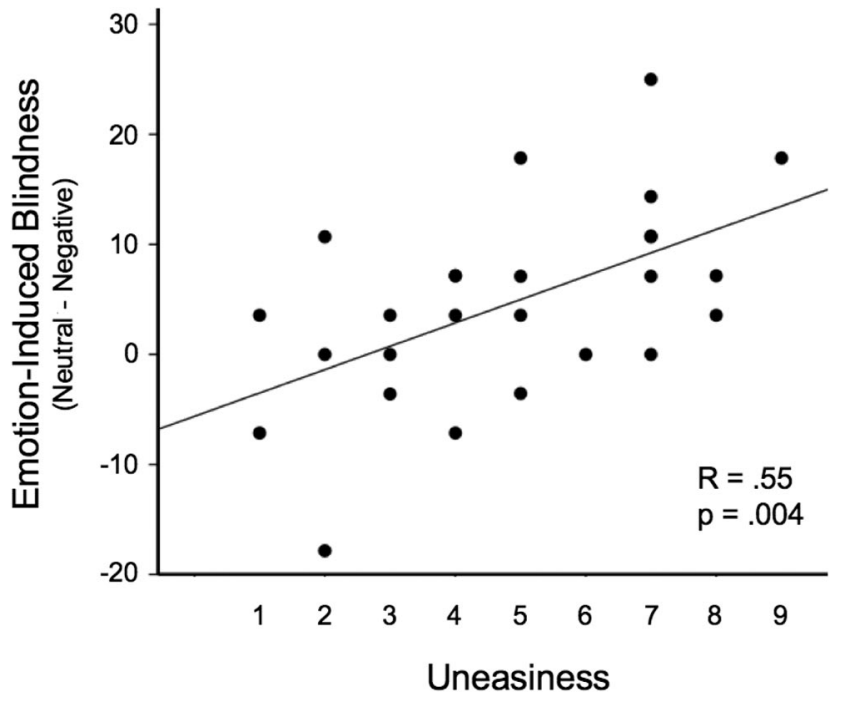

Figure 3. Data from Experiment 2. As in the first experiment, during the time that male partners were rating the attractiveness of other women, the degree to which negative emotional distractors elicited emotion-induced blindness among the female partners was significantly correlated with the degree to which they later rated themselves as having been uneasy about their male partner looking at other women.

induced blindness, $r=.539, p=.005$. (Indeed, there appeared to be no correlation at all between emotion-induced blindness during the landscape-rating condition and unease, $r=.113, p=.584$.) We also conducted a regression analysis incorporating global BIS, relationship length, and ratings of unease, which indicated that both level of unease about the male partner's task $(\beta=.54, t=$ $3.36, p=.004)$ and global threat sensitivity $(\beta=.55, t=3.46$, $p=.003$ ) were unique, independent, and significant predictors of emotion-induced blindness, whereas relationship length was not $(\beta=-.10, t=.62, n s)$. A similar regression analysis indicated that level of unease about the male partner's task also remained a significant predictor of emotion-induced blindness $(\beta=.53, t=$ $3.17, p=.006)$, independent of relationship threat sensitivity $(\beta=$ $.53, t=3.20, p=.005)$ and relationship length $(\beta=-.12, t=$ $.70, n s)$. It is curious that global- and relationship-threat sensitivity emerged as significant predictors in this experiment but not in Experiment 1; possible explanations are that this discrepancy is simply a consequence of testing different samples in the two experiments or a consequence of somewhat lower reliability of the BIS and RTS scales in Study 1. Notably, this discrepancy makes it all the more striking that the predictive power of self-rated unease should be so consistent in both cases.

\section{General Discussion}

In two separate experiments, we found strong associations between the degree to which people felt threatened by a social context and the degree to which task-irrelevant, negative emotional distractors grabbed attention and impaired visual awareness of targets. This finding addresses two questions simultaneously: (a) can emotion-induced blindness be modulated by an individual's emotional state?; and (b) can interpersonal factors-likely operating through their impact on emotional state- have such pervasive effects as to permeate processes involved in conscious perception? The answer to both questions appears to be yes.

Notably, although one reasonable prediction would have been that a heightened state of anxiety would increase general distractibility, in both experiments self-reported unease only correlated with performance decrements induced by distractors typically classified as emotionally negative, not with performance in neutral, erotic, or baseline conditions. This is despite the fact that the erotic distractors contained content evocative of intimacy and relationship threat and might-in the context of having one's partner rate the attractiveness of one's potential romantic rivals - have themselves taken on negative valence. Future experiments should continue to explore the potential specificity between emotional state and spontaneous attention to distractors. Furthermore, unease about having one's romantic partner rate the attractiveness of other women correlated with emotion-induced blindness only during the time that he was doing so, not when he was rating the attractiveness of landscapes. This is important, as it suggests that heightened sensitivity to emotional distractors in these experiments was a function of the effectiveness of the relationship threat manipulation rather than simply a function of a more general association between trait anxiety and a stronger bias to attend to emotional information. Underscoring this point, the relationship between self-rated unease and emotion-induced blindness remained strong and significant even when factored into a model that accounted for global and relationship-specific trait sensitivity to threat.

It is worth noting that although our a priori design choices allowed us to uncover correlations between the effectiveness of the relationship threat manipulation and emotion-induced blindness, these same choices possibly hindered our ability to reveal potentially interesting main effects. For example, in Experiment 2 we found no main effect or interactions involving the attentional set manipulation; it is possible that this manipulation might have had more impact had we counterbalanced the order of the specific- and nonspecific attentional set conditions. However, based on our anticipation of a strong role for individual differences in sensitivity to the relationship threat manipulation, we strategically chose to hold the order of conditions constant so as better to allow these individual differences to emerge. Future research might pursue the opposite tactic, more heavily emphasizing the main effects of the manipulations at the potential cost of being able to account for individual differences.

In the current experiments, it was always the female partner who performed the emotion-induced blindness task while her partner rated the attractiveness of pictures. This was not because of assumptions regarding sex differences but was instead motivated by the need to test the hypothesized effect in a relatively homogenous population (i.e., to eliminate as much noise as possible in ratings of general unease). It is an open question as to whether male participants would show the same effect if the roles were reversed; it may be that even a stronger relationship would emerge in such a scenario. Another question-given that romantic relationship quality predicts the degree to which threat-activated brain regions are calmed when holding a partner's hand (Coan et al., 2006) - is whether couple-to-couple individual differences moderate the influence of the partner's rating task on emotion-induced blindness. We did find some evidence, in Experiment 2, that trait-like differences in female partner sensitivity to relationship threat predicted emotion-induced blindness when participants' male partners were 
rating the attractiveness of potential romantic competitors, independent of self-rated unease. This provides more reason to suggest that relationship-related individual differences and features of the current relational context may indeed play a role in predicting visual attention, but such a claim as yet awaits further confirmation.

The language of social relationships is filled with visual metaphor: a person, for example, "sees" another's inner beauty but can be "blinded" by love or jealousy. Now, it turns out that such turns of phrase connect to reality perhaps more concretely than might have been expected. The influence of social emotions - known to affect moods, behaviors, and physical health - appears to permeate so deeply as to affect processes involved in visual awareness. Given the temptation to frame these findings specifically in terms of relationship threat, it is important to note that this manipulation was potentially one of several that might have a similar impact by virtue of increasing levels of anxiety and unease. That said, although lovers, poets, and philosophers may debate whether indeed love is blinding, the present evidence suggests that -in at least some situations-jealousy is.

\section{References}

Berscheid, E. (1983). Emotion. In H. H. Kelley, E. Berscheid, A. Christensen, J. H. Harvey, T. L. Huston, G. Levinger, ... D. R. Peterson (Eds.), Close relationships (pp. 110-168). New York: Freeman.

Bradley, M. M., Codispoti, M., Sabatinelli, D., \& Lang, P. J. (2001). Emotion and motivation II: Sex differences in picture processing. Emotion, 1, 300-319.

Brainard, D. H. (1997). The psychophysics toolbox. Spatial Vision, 10, 433-436.

Carver, C. S., \& White, T. (1994). Behavioral inhibition, behavioral activation, and affective responses to impending reward and punishment: The BIS/BAS scales. Journal of Personality and Social Psychology, 67, 319-333.

Chun, M. M., \& Marois, R. (2002). The dark side of visual attention. Current Opinion in Neurobiology, 12, 184-189.

Cloninger, R. C., Przybeck, T. R., \& Svrakic, D. M. (1991). The tridimensional personality questionnaire: U.S. normative data. Psychological Reports, 69, 1047-1057.

Coan, J. A., Schaefer, H. S., \& Davidson, R. J. (2006). Lending a hand: Social regulation of the neural response to threat. Psychological Science, 17, 1032-1039.

Fox, E., Russo, R., Bowles, R., \& Dutton, K. (2001). Do threatening stimuli draw or hold visual attention in subclinical anxiety? Journal of Experimental Psychology: General, 130, 681-700.

Lang, P. J., Bradley, M. M., \& Cuthbert, B. N. (2001). International affective picture system (IAPS): Instruction manual and affective ratings (Tech. Rep. No. A-5). Gainesville: University of Florida, Center for Research in Psychophysiology.

Laurenceau, J.-P., Kleinman, B. M., Kaczynski, K. J., \& Carver, C. S. (in press). Assessment of relationship-specific incentive and threat sensitivities: Predicting satisfaction and affect in adult intimate relationships. Psychological Assessment.

Mack, A., \& Rock, I. (1998). Inattentional blindness. Cambridge, MA: MIT Press.

Meng, X. L., Rosenthal, R., \& Rubin, D. B. (1992). Comparing correlated correlation coefficients. Psychological Bulletin, 111, 172-175.

Most, S. B., Chun, M. M., Johnson, M. R., \& Kiehl, K. A. (2006). Attentional modulation of the amygdala varies with personality. NeuroImage, 31, 934-944.

Most, S. B., \& Jungé, J. A. (2008). Don’t look back: Retroactive, dynamic costs and benefits of emotional capture. Visual Cognition, 16, 262-278.

Most, S. B., Scholl, B. J., Clifford, E., \& Simons, D. J. (2005). What you see is what you set: Sustained inattentional blindness and the capture of awareness. Psychological Review, 112, 217-242.

Most, S. B., Simons, D. J., Scholl, B. J., Jimenez, R., Clifford, E., \& Chabris, C. F. (2001). How not to be seen: The contribution of similarity and selective ignoring to sustained inattentional blindness. Psychological Science, 12, 9-17.

Most, S. B., Smith, S. D., Cooter, A. B., Levy, B. N., \& Zald, D. H. (2007). The naked truth: Positive, arousing distractors impair rapid target perception. Cognition \& Emotion, 21, 964-981.

Most. S. B., Chun, M. M., Widders, D. M., \& Zald, D. H. (2005). Attentional rubbernecking: Cognitive control and personality in emotion-induced blindness. Psychonomic Bulletin and Review, 12, 654 661.

Neisser, U., \& Becklen, R. (1975). Selective looking: Attending to visually specified events. Cognitive Psychology, 7, 480-494.

Pelli, D. G. (1997). The VideoToolbox software for visual psychophysics: Transforming numbers into movies. Spatial Vision, 10, 437-442.

Reis, H. T., \& Collins, W. A. (2004). Relationships, human behavior, and psychological science. Current Directions in Psychological Science, 13, 233-237.

Reis, H. T., Collins, W. A., \& Berscheid, E. (2000). The relationship context of human behavior and development. Psychological Bulletin, $126,844-872$.

Simons, D. J., \& Chabris, C. F. (1999). Gorillas in our midst: Sustained inattentional blindness for dynamic events. Perception, 28, 1059-1074.

Simpson, J. A., Ickes, W., \& Blackstone, T. (1995). When the head protects the heart: Empathetic accuracy in dating relationships. Journal of Personality and Social Psychology, 69, 629-641.

Received September 10, 2008

Revision received September 2, 2009

Accepted September 26, 2009 